\section{The networking cytoskeleton}

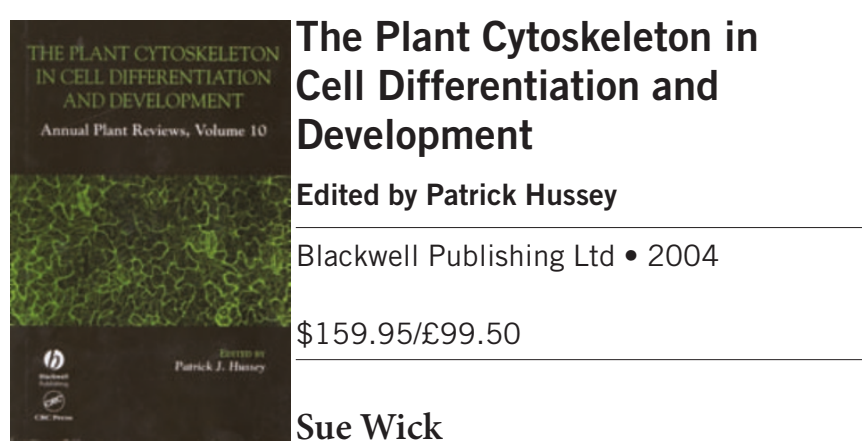

What a difference a few decades makes! Back in the 1980s and early 1990 s, our knowledge of the plant cytoskeleton relied almost entirely on basic - sometimes brute-force - biochemistry of isolated molecules, microscopy of fixed cells and immunochemistry. In 2005, we now have the advantage of a wealth of new techniques and data: imaging tools that can be used to examine live cells expressing fluorescently tagged proteins, post-genomic sequence information from the Arabidopsis thaliana genome project, sophisticated molecular genetics and the appearance of several new laboratories working on the plant cytoskeleton.

The Plant Cytoskeleton in Cell Differentiation and Development, edited by Patrick Hussey, captures the excitement and advances made in the field after the explosive growth of the past several years. The 10 book chapters are assembled into three parts. The first two chapters deal with the principal plant cytoskeletal molecules, tubulin and actin, and the many associated proteins that regulate their nucleation, polymerization status and behaviour. The second part - chapters 3 to 6 - includes fundamental cellular processes that rely on the cytoskeleton, such as mitosis and organelle movements. The last part - chapters 7 to 10 - describes the cytoskeletal involvement in the differentiation and functioning of models such as root hairs and stomatal guard cells.

Of course, the cytoskeleton should not be seen as operating in isolation - indeed, the direct differentiation and development of individual cells, and of course of entire plants, involves a complex array of inter-related processes. These processes require cells first to sense internal stimuli (such as hormone and ion gradients) and also external factors (such as light quality, gravity, moisture and ion levels). Cells can then respond to the stimuli by establishing and interpreting polarity and communicating signal information within and between themselves. So, the cytoskeleton often finds itself in the middle of a web of pathways: chemical signals can influence cytoskeletal dynamics and organization, which in turn can modulate further transduction of the signal. In keeping with our current understanding of how the cytoskeleton is enmeshed in cell development, the theme of signal-transduction pathways and their interactions with the cytoskeleton is woven through several chapters.

Sue Wick is in the Department of Plant Biology, University of Minnesota, 250 BioSciences Center, 1445 Gortner Avenue, St Paul, MN, 55108-1095, USA.

e-mail: swick@cbs.umn.edu
Indeed, one of the greatest strengths of the book is that several authors have gone beyond the idea of considering the cytoskeleton in isolation, and have presented a larger picture of the cytoskeleton as being at the crossroads of important biochemical and vesicle trafficking events. A whole array of components swirl through the book, as they do through the plant cell, including protein kinase and phosphatase cascades, small GTPase switches that coordinate pathways, phosphoinositides, $\mathrm{Ca}^{2+}$-calmodulin, 14-3-3 proteins that activate signal-transduction pathways and the still-controversial cyclic nucleotides. Aspects of cell-wall extensibility find a mention in all chapters that deal with growth processes, as well as in Chapter 6, which is devoted to the cell wall, its mechanical and development-shaping properties, and its role as a key link in signal transduction. A few authors go further afield and give so much detail about other material that the cytoskeleton ends up being a minor player. For example, there is coverage in Chapter 6 of the phenomenal complexity of the cell-wall polysaccharides, proteins and aromatic substances, and there is a large amount of detail given in Chapter 4 on the cyclins and various protein kinases that regulate the cell cycle. In general, however, the chapters taken together are a strong rendition of the breadth of where the plant cytoskeleton fits in relation to the rest of the cell and its activities.

This book — which was written in 2004 - is as up-to-date as any bound book is likely to be, covering research carried out up to and including 2002, with a sprinkling of citations for 2003. It will be a useful reference for graduate students coming into the field, as well as for seasoned researchers. Its highly detailed table of contents facilitates finding specific topics, and the tables and diagrams that are used to organize complex material are particularly useful for any reader trying to assimilate the wealth of information contained here. For the most part, the chapters complement each other well. One confusing detail, however, is in using the term 'phragmosome' to mean two totally different things in Chapters 5 and 6. For most plant-cell biologists, the definition of phragmosome is the one given in Chapter 5 - a planar array of membranous transvacuolar strands that suspend the premitotic and mitotic nucleus.

The fact that elements of the cytoskeleton and of signal-transduction pathways show high evolutionary conservation across biological kingdoms speaks to their centrality in eukaryotic life. As pointed out by the editor, the real excitement for plant biologists lies in how those elements have evolved together to allow for features and processes that are unique to the life of plants. When reading this book, one feels as if the plant cytoskeleton has finally come of age. Having been the poor cousins of the animal and yeast cytoskeleton research community for so many years, plant cytoskeleton researchers have now gathered strength and data - a situation that may be viewed as both a blessing and a curse. On the one hand, there are now so many more pieces of the incredibly complex picture of how the cytoskeleton, in response to cues, participates to direct numerous aspects of plant-cell development. On the other hand, there is the likelihood that the growing momentum of research in this area will soon make this book out of date. 